\title{
PLAFIR: aplicativo web para planejamento financeiro rural
}

\author{
Luciano Lizzoni \\ Aldi Feiden \\ Armin Feiden \\ Universidade Estadual do Oeste do Paraná - Unioeste, Brasil
}

CASE REPORT

\begin{abstract}
Resumo
Objetivo. A diversificação rural é um meio para que agricultores familiares prosperem ou até mesmo não pereçam. Contudo, decidir por uma nova atividade rural pode se tornar um trabalho árduo. O objetivo deste trabalho é desenvolver um Sistema de Informação que auxilie o agricultor na tomada de decisão por meio de indicadores financeiros.

Método. Foi aplicado um questionário a vinte agricultores familiares do Oeste Paranaense, com abordagem quantitativa, e realizada uma pesquisa bibliográfica e documental para verificar os Sistemas de Informação disponíveis no mercado. Posteriormente, foi elaborado um projeto de software, utilizando elementos da UML, e como framework de desenvolvimento o ASP.NET Core MVC 1.1, com banco de dados PostgreSQL 9.5. A IDE utilizada para desenvolvimento foi o Microsoft Visual Studio 2015 Community Edition. Os testes foram realizados em ambiente Linux com Ubuntu 14.04, Apache 2 e navegador Chrome 55.

Resultados. A implementação do projeto de software resultou no aplicativo denominado de Planejamento Financeiro Rural (Plafir). Este é um aplicativo web que auxilia na tomada de decisão, quando o agricultor decide investir em uma nova atividade rural. Com base em modelos financeiros pré-cadastrados no sistema por técnicos agrícolas e uma tabela de insumos com preços atualizados, o agricultor pode selecionar determinado modelo, simular a produção e conferir os resultados financeiros.

Conclusões. A agricultura familiar está desprovida de Sistemas de Informação para a tomada de decisão e o aplicativo Plafir atende ao objetivo proposto neste estudo, contribuindo para a seleção de uma nova atividade rural.
\end{abstract}

Palavras-chave

Agricultura familiar; Diversificação rural; Orçamento; Sistema de informação; Tomada de decisão

\section{PLAFIR: web application for rural financial planning}

\section{Abstract}

Objective. The rural diversification is a way for family farmers to prosper or even to perish. However, deciding a new rural activity can become hard work. The objective of this work is creating an Information System that helps the farmer in decision making, through financial indicators.

Method. A questionnaire was applied to 20 family farmers of Oeste Paranaense with a quantitative approach, and a bibliographical and documentary research was carried out to verify Information Systems available. Subsequently, a software project was developed using UML elements and the ASP.NET Core MVC 1.1 development framework with PostgreSQL 9.5 database. The IDE used for development was the Microsoft Visual Studio 2015 Community Edition. The tests were performed in a Linux environment with Ubuntu 14.04, Apache 2, and Chrome 55 browser.

Results. The implementation of the software project resulted in the nominated application of Rural Financial Planning (Plafir). Plafir is a web application that assists in decision making when the farmer decides to invest in a new rural activity. Based on financial models pre-registered in the system by agricultural technicians, and a table of inputs with updated prices, the farmer can select a particular model, simulate the production, and check the financial results.

Conclusions. Family farming is lacking Information Systems for decision making, and the application Plafir meets the objective proposed in this study, contributing to the decision making of a new rural activity.

Keywords

Budget; Decision making; Family farming; Information system; Rural diversification 


\section{Introdução}

O desenvolvimento sustentável tem sido debatido nos últimos anos como um meio de crescimento econômico, porém sem destruição do meio ambiente e com garantia de desenvolvimento social para as próximas gerações. No âmbito rural, um dos atores que dependem de práticas sustentáveis como uma via de manutenção de renda, e uma forma de garantir a sua existência, é o agricultor familiar.

A agricultura familiar ganha notoriedade pela expressiva contribuição na alimentação global. Países com o índice de desenvolvimento humano elevado possuem presença acentuada da agricultura familiar, pois combate a pobreza, reduz a fome e seu meio de conduta segue os princípios da sustentabilidade (GUANZIROLI et al., 2001; FAO, 2014; AIAF, 2014).

Uma das características predominantes da agricultura familiar é a diversificação rural. Este meio é uma alternativa para o crescimento econômico, pois promove oportunidade de diferenciação, aumento de renda e qualidade de vida, além de manter estabilidade financeira perante as oscilações econômicas. Entretanto, decidir por uma nova atividade rural depende da análise de vários fatores. Muitas vezes, o agricultor familiar não possui conhecimento suficiente para a tomada de decisão, sendo dependente de terceiros, o que inibe seu crescimento.

Um dos aspectos cruciais na tomada de decisão para a diversificação é a projeção financeira. Planejar o orçamento da produção provê subsídios para a decisão estratégica do negócio. Compreender a quantidade de insumos, a mão de obra necessária e a projeção de resultado são elementos importantes no momento de decidir. Porém, diversos elementos compõem um planejamento financeiro, o que requer habilidades para construir e analisar os resultados.

Assim, na era da Internet, a Tecnologia da Informação é uma grande aliada na tomada de decisão. Os Sistemas de Informação proporcionam agilidade no tratamento de dados, segurança na troca e armazenamento destes e confiabilidade nos resultados. Ainda, geram relatórios gerenciais que apontam as deficiências do negócio. Um Sistema de Informação, na Internet, tem seu funcionamento independente de instalação ou manutenção. $O$ acesso está disponível a partir de vários lugares e com qualquer dispositivo tecnológico. Novas tecnologias, como o caso do .NET Core, software proprietário desenvolvido pela Microsoft, possibilitam criar aplicações web de maneira rápida e independente de plataforma.

Entretanto, a agricultura familiar está carente de soluções em Sistemas de Informação para a tomada de decisão. Muitos dos softwares disponíveis no mercado são inadequados ou pela sua complexidade, ou por não atenderem ao problema proposto.

Inserido nesta ótica, o objetivo deste estudo é implementar um Sistema de Informação que auxilie na tomada de decisão, quando o agricultor decide investir em uma nova atividade rural por meio de indicadores financeiros. $A$ pesquisa de atos normativos, pelos servidores da Aneel, constitui um ato decisório, em que o usuário diante da interface de busca, identifica a sua necessidade de informação e utilizando seus conhecimentos prévios sobre o sistema de busca e sobre o tema a ser pesquisado, traça uma estratégia de pesquisa, utilizando alguns descritores a fim de encontrar o que necessita.

\section{Revisão bibliográfica}

\subsection{Desenvolvimento rural sustentável por meio da agricultura familiar}

O desenvolvimento sustentável parte da concepção de satisfazer as necessidades atuais da geração presente, sem afetar as próximas gerações. A consolidação da sustentabilidade ocorre na presença do equilíbrio entre as dimensões econômica, social e ambiental (GUZMÁN, 1998; SACHS, 1993).

Um dos atores que podem utilizar-se dos princípios do desenvolvimento sustentável, no âmbito rural, são os agricultores familiares. Considera-se agricultor familiar aquele cuja propriedade tenha predomínio da mão de obra familiar, com área que não ultrapasse a quatro módulos fiscais. Ainda, que os moradores dependam da renda gerada pela unidade produtiva, conforme Lei $n^{0} 11.326$, de 24 de julho de 2006 e a Portaria MDA $n^{\circ} 21$, de 27 de março de 2014. 
A agricultura familiar ganha evidência, ao analisar os dados do último Censo Agropecuário realizado no Brasil. Cerca de $70 \%$ da alimentação brasileira é proveniente da agricultura familiar, correspondendo a $84,4 \%$ de ocupação em todo o segmento rural (IBGE, 2006).

O Comitê Brasileiro publicou argumentos e dados sobre a agricultura familiar no mundo, e enfatiza cinco grandes motivos de apoiar a iniciativa: garantir a alimentação e acabar com a fome, gerar bem-estar, combater à pobreza, proteger a biodiversidade e contar com a mulher produtora (FAO, 2014).

Uma característica da agricultura familiar é a diversificação rural. Para sobreviver e melhorar o padrão de vida, os agricultores familiares constroem diversos bens e atividades. Estes promovem aumento de renda, fortalecimento em frente às oscilações econômicas, além de reduzirem a dependência (ELLIS, 2000; SCHNEIDER, 2010).

\subsection{Planejamento financeiro rural}

Diante da necessidade de diversificar, o planejamento é essencial para qualquer tipo de negócio, inclusive para a agricultura familiar, ao iniciar novas atividades rurais. Planejar é um processo contínuo e sistemático de tomar decisões, prevendo consequências futuras para, posteriormente, comparar resultados. Por isso, é importante planejar, no sentido de alertar quanto às mudanças na economia, hábitos de consumidores, avanços da tecnologia e demanda de produtos (SANTOS; MARION; SEGATTI, 2009).

Sob esse prisma, o planejamento operacional se destaca por trazer um conjunto de planos orçamentários que implicam a utilização de artefatos contábeis que projetam um resultado. As principais peças de um conjunto orçamentário são: área; produto; produtividade; preços de venda; impostos; mão de obra; insumos agrícolas; equipamentos; custos indiretos e despesas administrativas. É de fundamental importância que produtores façam orçamentos para sua produção de maneira que auxilie na decisão estratégia dos negócios (SANTOS; MARION; SEGATTI, 2009).

Um planejamento financeiro de determinada atividade rural pode ser aproveitado por vários agricultores, porém é necessário que cada um destes informe suas despesas, a área cultivada, o maquinário utilizado e as benfeitorias disponíveis. Por outro lado, os custos de produção são semelhantes. Custos são os gastos atrelados direta ou indiretamente à produtividade, tais quais: sementes, fertilizantes, defensivos e mão de obra. Já as despesas são gastos provenientes de vendas e demais despesas financeiras ou administrativas (MARION, 2014).

Por outro lado, o custo de produção é uma excepcional ferramenta de gestão, ao gerar informações que subsidiem a tomada de decisão dos produtores rurais. Os itens que compõem o custo de produção dividem-se em custo variável e fixo. O custo variável apresenta operações com máquinas, mão de obra, sementes, fertilizantes, agrotóxicos, irrigação, entre outros. Por outro lado, o custo fixo apresenta depreciações de benfeitorias, máquinas e implementos, exaustão do cultivo e seguros. Cabe salientar que, para compor o custo total, incluem-se renda sobre a terra e remuneração esperada sobre capital fixo (CONAB, 2010).

\subsection{Sistemas de informação na agricultura}

Realizar um planejamento financeiro sem o uso da Tecnologia da Informação pode se tornar um processo lento e suscetível a erros. Até mesmo o uso de planilhas eletrônicas exige um grande conhecimento técnico sobre informática e o assunto em estudo. Com o uso de Sistemas de Informação (SI), o agricultor despenderá menos do seu tempo, pois obterá dados de maneira instantânea, organizada e precisa (SANTOS; MARION; SEGATTI, 2009).

Além disso, os Sistemas de Informação controlam a entrada, processamento e saída de dados. Estes, armazenados digitalmente, oferecem possibilidade para posterior consulta. Entretanto, os SI vão além de aplicativos: são compostos por pessoas, processos de negócios e TI (LAUDON; LAUDON, 2010).

Há muitos agricultores que ainda desconhecem as vantagens da informática. Entretanto, a nova geração de trabalhadores já está habituada a dispositivos tecnológicos e se sentem confortáveis para utilizar a informática (MENDES; OLIVEIRA; SANTOS, 2011). No Brasil, em 2014, cerca de 95,4 milhões de pessoas tiveram acesso 
à Internet, um crescimento de 11,4\% em relação ao ano anterior. Pessoas de 15 a 19 anos de idade apresentam maior participação, com cerca de $81 \%$ (IBGE, 2015).

Os Sistemas de Informação baseados na Internet são considerados ampliadores do negócio, pois ajudam a todos os tipos de empresa na tomada de decisão e nos resultados. Um aplicativo na Internet está disponível a todos, independentemente da plataforma ou dispositivo de acesso (O'BRIEN, 2004).

Quando as soluções em software disponíveis no mercado são inadequadas, devido à complexidade de manuseio ou até mesmo à inexistência de solução para determinada situação, é necessário que Analistas de Sistemas elaborem projetos de softwares para novas ferramentas que auxiliem agricultores.

\subsection{Projeto de software}

A primeira parte no desenvolvimento de um software é definir e entender o problema, ou seja, identificar suas causas, objetivos de solução e requisitos de informação. Depois de organizar estes dados, o próximo passo é buscar alternativas existentes que se adaptam ao problema. Caso o resultado da busca não seja qualificado, inicia-se, neste momento, a concepção de um projeto de software. Uma ferramenta que auxilia na fase de concepção é a UML (LAUDON; LAUDON, 2010).

A UML (Unified Modeling Language ou Linguagem de Modelagem Unificada) trata de uma linguagem visual criada com o propósito de modelar sistemas orientados a objetos. Ela possui elementos gráficos que auxiliam no desenvolvimento de um projeto. Por meio destes elementos, é possível visualizar diversas perspectivas de um sistema. A UML é independente de linguagem de programação e de processos de desenvolvimento de software (BEZERRA, 2015). Auxilia na definição das características de um software como seus requisitos, atores, processos e infraestrutura (GUEDES, 2014). Com os elementos da UML é possível obter um esboço do projeto, pois suas notações gráficas ajudam a descrever um projeto de software (FOWLER, 2014).

O primeiro passo para a concepção de um Sistema de Informação é o levantamento de requisitos. Este processo consiste em identificar o problema e definir as necessidades para solucionar este problema. $O$ levantamento é realizado por meio de um estudo exploratório e resulta em um documento de requisitos do sistema. Tais requisitos podem ser funcionais, que definem funcionalidades do sistema, ou não funcionais, que definem características de qualidade referentes à confiabilidade, desempenho, portabilidade, segurança e utilização (BEZERRA, 2015).

A etapa da análise de requisitos envolve detalhar os eventos do negócio, as atividades, os produtos a serem gerados, as entidades envolvidas, entre outros. O tempo reduzido no desenvolvimento desta etapa resultará em maior custo na manutenção e correção no Sistema de Informação (SORDI; MEIRELES, 2010).

Por outro lado, os atores são elementos que representam papéis de diversos usuários e podem interagir com funcionalidades do sistema (GUEDES, 2014). Os atores de negócios podem ser pessoas, organizações ou outros sistemas. Eles interagem com os casos de uso e diagramas de sequência (WAZLAWICK, 2015).

O diagrama de sequência, assim como o caso de uso expandido, procura demonstrar a série de eventos de um processo e a relação entre atores e objetos envolvidos no sistema. Por representar graficamente um caso de uso de modo detalhado, permite visualizar o fluxo de dados, desde a primeira interação do usuário até a apresentação final dos resultados (GUEDES, 2014). O objetivo é mostrar as interações entre atores e sistema. O diagrama possui elementos gráficos que simbolizam a troca de mensagens, com finalidade de compreender a linha de execução de um processo (BEZERRA, 2015).

Especificar um novo Sistema de Informação (SI) significa compreender a demanda dos usuários do SI, além de discutir e gerar uma documentação apropriada para que todos os envolvidos no projeto possam entendê-lo. Para tanto, devido à importância desta fase, há um profissional com a competência técnica apropriada para desenvolver um projeto de software: o analista de sistemas (SORDI; MEIRELES, 2010). 


\subsection{Banco de dados}

Os dados têm se tornado cada vez mais essenciais para as organizações sobreviverem e prosperarem. Estas devem manter seus dados à disposição, para tomar decisões quando necessário. Pode-se argumentar que o objetivo final de todos os Sistemas de Informação é ajudar as empresas a usar a informação como um recurso da organização. No centro de todos esses sistemas, estão a coleta, armazenamento, agregação, manipulação, disseminação e gerenciamento de dados (CORONEL; MORRIS, 2016).

Os dados são onipresentes (abundantes, globais em toda parte) e penetrantes (inescapáveis, prevalentes, persistentes). Desde o nascimento até a morte, as pessoas geram e consomem enormes quantidades de dados. Nesse sentido, os bancos de dados são a melhor maneira de armazenar e gerenciar dados, pois os tornam persistentes e compartilháveis de forma segura (CORONEL; MORRIS, 2016).

Um banco de dados amplamente conhecido é o PostgreSQL. Possui uma ampla gama de plataformas e boa reputação nos aspectos de desempenho, estabilidade e recursos avançados. O PostgreSQL é um dos mais antigos projetos de código aberto, totalmente livre para usar e desenvolvido por uma comunidade mundial muito diversificada (RIGGS, 2015).

O PostgreSQL é um sistema de gerenciamento que começou como um projeto de pesquisa na Universidade da Califórnia, em Berkeley. Ao permitir escrever procedimentos e funções em vários idiomas de programação, em sua instalação-padrão se encontra o aplicativo pgAdmin, uma ferramenta de manipulação com a qual é possível explorar as tabelas, campos, dados, procedimentos, extensões, entre outros (OBE; HSU, 2014).

\subsection{NET Core}

A plataforma .NET Core é uma evolução: o .NET 1.0 foi lançado em 2002 e, em 2016, está na versão 4.6. Visto que a versão .NET Core foi totalmente redesenhada, ela não seguiu a numeração de sua versão ancestral e acabou somando o termo "Core" em seu nome, com a atual versão 1.1 em 2017. O .NET Core é baseado em pequenas unidades e pacotes NuGet, arquivos compactados que contêm códigos compilados com determinadas funcionalidades e podem ser encontrados e atualizados facilmente. Isto porque a plataforma .NET Core é modular e permite adicionar bibliotecas de terceiros, conforme a conveniência. Permite, ainda, executar aplicações em ambiente Windows, Linux e Mac, além de ser um projeto de código aberto (NAGEL, 2016).

Já o ASP.NET Core MVC é um framework de desenvolvimento de aplicações web, criado pela Microsoft. Esta plataforma combina o conceito de arquitetura modelo-visão-controlador (MVC), técnicas de desenvolvimento ágil e funciona sobre a plataforma .NET Core. Também permite construir aplicações de uma forma simples e elegante, utilizando CSS (Cascading Style Sheets) e bibliotecas como jQuery e Bootstrap. Sua API proporciona inovações como o conceito de await, extensão de métodos, expressões em lambda, tipos dinâmicos e anônimos, além de expressões em LINQ (Language Integrated Query) (FREEMAN, 2016).

O ASP.NET Core MVC possui atributos de métodos para facilitar a configuração de funções no controlador. Atributos como HttpGet, HttpPost ou anotações de permissão de usuário, como Authorize e AllowAnonymous, facilitam o desenvolvimento web. Outra vantagem são as chamadas TagHelpers. O desenvolvedor pode criar suas próprias tags HTML, porém atribuindo funcionalidades próprias. Desta maneira, a programação visual torna-se mais elegante, sem misturar códigos de programação (CHAMBERS; PAQUETTE; TIMMS, 2017).

Para um melhor rendimento no desenvolvimento e diagramação de telas, há as IDEs (Integrated Development Environment ou Ambiente Integrado de Desenvolvimento), disponíveis para trabalhar, tanto com ASP.NET Core MVC, quanto com o MonoDevelop, o JetBrains Rider e o Microsoft Visual Studio Code. Além disso, é possível codificar na nuvem com um navegador, que simula uma IDE, chamado Cloud9. A vantagem em utilizar a versão Microsoft Visual Studio Community Edition é o número de ferramentas agregadas, que agilizam o desenvolvimento, além das funções para instalar e atualizar bibliotecas, do depurador de código, publicador de aplicações e emulação de navegador, entre outros. Não é preciso memorizar linhas de comando para compilar, executar ou publicar a aplicação. Esta IDE permite, ainda, instalar templates que criam o esboço de uma aplicação, com validação de usuários e desenvolvimento na estrutura MVC (PRICE, 2016). 


\section{Materiais e métodos}

Inicialmente, foi realizado um levantamento do perfil dos agricultores familiares por meio de uma pesquisa exploratória com abordagem quantitativa, utilizando questionários como instrumento de coleta de dados. 0 período da coleta foi em agosto de 2016 e contempla uma amostragem aleatória de 20 agricultores familiares, distribuídos em seis municípios da região Oeste do Paraná, sendo: Marechal Cândido Rondon (9), Quatro Pontes (4), Nova Santa Rosa (2), Entre Rios do Oeste (3), Toledo (1) e Mercedes (1). Todos eles possuem como mão de obra predominante a familiar e área até quatro módulos fiscais, ou seja, até 72 hectares segundo IAP (2016).

Um segundo levantamento foi realizado, de caráter bibliográfico e documental com abordagem qualitativa, no mesmo período. O objetivo deste levantamento foi verificar a existência de Sistemas de Informação disponíveis no mercado, que permitem executar um planejamento financeiro de atividades rurais, porém sem necessidade prévia de lançamento de dados históricos de produção.

Com base nos dados coletados, foram elaborados os requisitos do sistema. Estes contemplam necessidades funcionais do aplicativo como também características referentes à interface, utilização e desempenho, conforme Quadro 1.

Quadro 1. Relação de requisitos funcionais e suplementares do projeto.

Requisitos do sistema

F1 Simular planejamento financeiro em tempo real

Possibilitar ao agricultor selecionar uma atividade rural de interesse (baseado em modelos já cadastrados no sistema), visualizar o planejamento financeiro, manipular os dados e recalcular de maneira automática os indicadores financeiros.

O planejamento deve apresentar o valor da receita bruta estimada e o detalhamento dos custos variáveis (mão de obra, operações com máquinas, sementes, fertilizantes, defensivos, energia etc.). Estes agrupados conforme necessidade (receita, insumos, serviços etc.). O resultado deve apresentar o total de custos, o custo unitário e a margem bruta.

\section{F2 Registrar planos}

Permitir incluir, consultar, alterar e excluir o planejamento financeiro do agricultor.

\section{F3 Registrar modelos}

Permitir incluir, consultar, alterar e excluir modelos de planejamento financeiro elaborados por técnicos agrícolas.

F4 Registrar itens financeiros e histórico de preços

Permitir incluir, consultar, alterar e excluir itens que compõem o planejamento financeiro, além de seus preços.

F5 Apresentar evolução de preços de itens por meio de um gráfico

Permitir informar um período inicial e final de consulta para obter uma linha de variação de preços.

S1 Aplicativo web

O aplicativo deverá executar em ambiente web por meio de navegador de Internet.

$\underline{\text { S2 Website responsivo }}$

As telas do aplicativo deverão se ajustar conforme resolução adotada pelo usuário.

Fonte: Dados da pesquisa (2016)

Os requisitos funcionais foram utilizados ao longo do projeto para nortear o desenvolvimento das funcionalidades do aplicativo. Por outro lado, os requisitos suplementares são regras que colaboraram para a definição da tecnologia empregada.

Foram identificados os seguintes atores envolvidos no projeto: o usuário anônimo, o agricultor e o técnico agrícola. Os atores interagem com as funcionalidades do sistema e possuem papéis diferenciados. Devido a 
isto, foi criada a relação de permissões de acesso ao aplicativo, de acordo com os requisitos funcionais do sistema, conforme o Quadro 2.

Quadro 2. Relação de permissões de acesso ao sistema.

\begin{tabular}{|l|c|c|c|c|c|}
\hline Ator & $\begin{array}{l}\text { F1 Simular } \\
\text { planejamento }\end{array}$ & $\begin{array}{l}\text { F2 Registrar } \\
\text { planos }\end{array}$ & $\begin{array}{l}\text { F3 Registrar } \\
\text { modelos }\end{array}$ & $\begin{array}{l}\text { F4 Registrar itens } \\
\text { e preços }\end{array}$ & $\begin{array}{l}\text { F5 Gráfico de } \\
\text { preços }\end{array}$ \\
\hline Usuário anônimo & $\operatorname{Sim}$ & Não & Não & Não & Sim \\
\hline Agricultor & $\operatorname{Sim}$ & $\operatorname{Sim}$ & Não & Não & Sim \\
\hline Técnico Agrícola & $\operatorname{Sim}$ & $\operatorname{Sim}$ & Sim & Sim & Sim \\
\hline
\end{tabular}

Fonte: Dados da pesquisa (2016)

Com esta relação, tornou-se possível determinar a necessidade de diferenciar o usuário conectado e os aspectos visuais que se modificam conforme o perfil deste usuário. Por outro lado, o usuário anônimo pode ser qualquer pessoa que acesse o aplicativo, porém sem efetuar o login no sistema.

$\mathrm{Na}$ sequência, foram especificados os casos de uso para as funcionalidades. Há três cadastros com as operações de CRUD (acrônimo para operações de visualizar, excluir, inserir e alterar): planos financeiros, modelos de planejamento e itens, além dos preços. O principal caso de uso que define a funcionalidade do aplicativo é "D1 Simular planejamento financeiro em tempo real". Para este caso, elaborou-se o diagrama de sequência no aplicativo web Draw.io, conforme Figura 1.

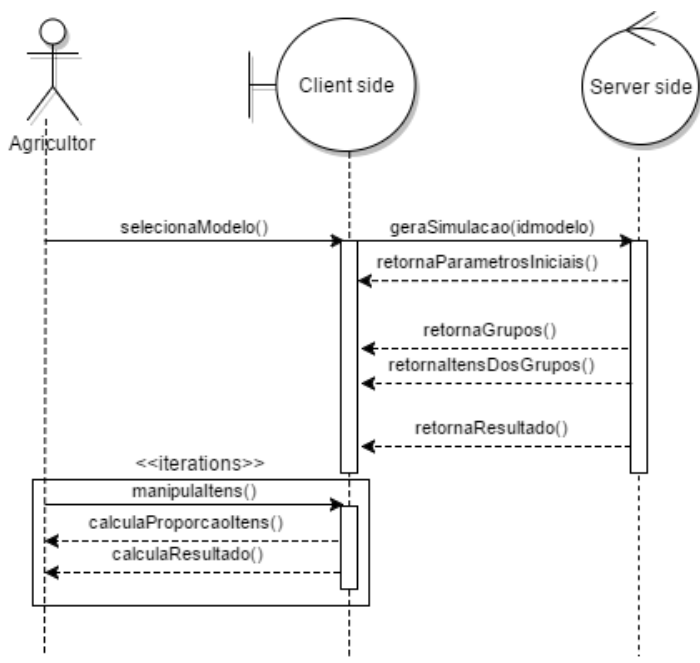

Figura 1. Diagrama de sequência "D1 Simular planejamento financeiro em tempo real"

Fonte: Dados da pesquisa (2016)

Este diagrama mostra que somente o primeiro evento faz a requisição para o servidor. Posteriormente, a interação do usuário é feita ao lado do cliente. Logo, esta característica determinou o uso de tecnologias que permitem tal ação.

Após o levantamento e a análise dos requisitos e a definição dos casos de uso, foi construído um diagrama de classes no aplicativo web Draw.io. Este apresenta o mapeamento das entidades do banco de dados e as relações com outras entidades, conforme a Figura 2. 


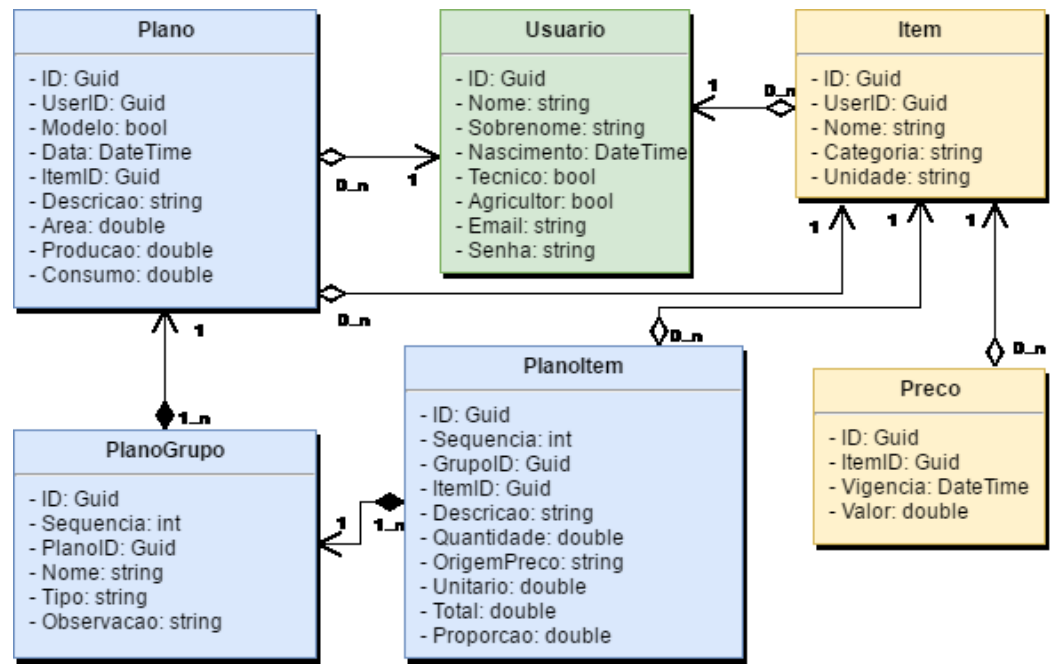

Figura 2. Diagrama de classes

Fonte: Dados da pesquisa (2016)

As tecnologias utilizadas neste projeto levaram em conta os requisitos e casos de uso. Considerando os requisitos $\mathrm{F} 1$, $\mathrm{S} 1$ e $\mathrm{S} 2$, além do diagrama $\mathrm{D} 1$, o aplicativo foi desenvolvido na plataforma .NET Core 1.1, com o framework ASP.NET Core MVC 1.1. Esta tecnologia permite o desenvolvimento com bibliotecas javascript (operações em client-side) e o renderizador de CSS Bootstrap (website responsivo). Os editores visuais, componentes de texto, data, valor e botões, utilizam a biblioteca javascript de código aberto KendoUI Core 2016.3.1103. Para a confecção do gráfico, foi utilizada a biblioteca javascript Charts.js 2.4.

Por outro lado, o banco de dados utilizado foi o PostgreSQL 9.5 junto de duas extensões: unaccent e uuid-ossp. O acesso ao banco se deu pela ferramenta de persistência Entity Framework Core 1.1, com o provider NpgsqIPostgreSQL 1.1. As classes descritas no diagrama representam o mapeamento das entidades do banco de dados.

No ambiente de desenvolvimento, a IDE utilizada foi o Microsoft Visual Studio 2015 Community Edition, na plataforma Windows. Este aplicativo possui funcionalidades agregadas, que auxiliam na criação de novos projetos. O template para a criação inicial do projeto foi o .NET Core 1.01 Preview 2, com opções de autenticação de usuário, utilizando o modelo MVC.

Por fim, o teste do aplicativo resultante, o server-side, foi em ambiente Linux, com o sistema operacional Ubuntu 14.04 e servidor web Apache 2. Já no client-side, foi utilizado o navegador Chrome 55.

\section{Discussão dos resultados}

A implementação do projeto de software resultou no aplicativo denominado de Planejamento Financeiro Rural (Plafir - http://www.plafir.com.br), que auxilia na tomada de decisão quando o agricultor decide investir em uma nova atividade rural. Com base em modelos financeiros pré-cadastrados no sistema por técnicos agrícolas e uma tabela de insumos com preços atualizados, o agricultor pode selecionar determinado modelo, simular a produção e conferir os resultados financeiros.

Os preços de insumos não são atualizados de forma automática. Conforme a necessidade, o técnico agrícola deverá fazer um levantamento dos preços na região, e registrá-los no Plafir. Inicialmente, o projeto estará disponível de forma gratuita aos agricultores da região Oeste do Paraná, de acordo com a região pesquisada.

A concepção do projeto de software se deu com os dados obtidos na pesquisa com os agricultores familiares e na pesquisa dos Sistemas de Informação disponíveis no mercado. Os principais resultados obtidos foram: $60 \%$ dos agricultores têm interesse em diversificar suas atividades; as atividades predominantes nas propriedades são o cultivo de milho e soja; ao decidir em qual atividade investir, $65 \%$ dos agricultores possuem dificuldade para levantar aspectos financeiros da atividade; somente $20 \%$ conhecem pessoas ou entidades que ajudam a decidir por uma nova atividade; $40 \%$ conhecem pessoas ou entidades que desenvolvem um planejamento financeiro; $100 \%$ possuem acesso à Internet e procuram aprimorar seu conhecimento para buscar melhorias na propriedade. 
A pesquisa ainda mostrou que $100 \%$ gostariam de uma ferramenta que fornecesse preços atualizados e $92 \%$ apreciariam uma ferramenta de simulação online. Referente aos Sistemas de Informação disponíveis no mercado, são poucos os que permitem simulação financeira sem necessidade prévia de lançamento de dados históricos de produção. Dos existentes, nenhum possui atualização de funcionalidades e os preços de insumos estão desatualizados.

Seguindo os requisitos do projeto, este aplicativo independe de instalação física e pode ser acessado por dispositivos conectados à internet, com resolução de tela distinta como laptops, tablets e smartphones. As telas se ajustam ao tamanho correspondente ao do dispositivo. Esta característica é proveniente dos requisitos S1 e S2.

Para deixar o aplicativo simplificado e intuitivo, a tela inicial do Plafir possui um editor, semelhante a sites de buscas como o Google. O agricultor pode buscar o modelo desejado sem precisar se identificar, conforme a Figura 3.

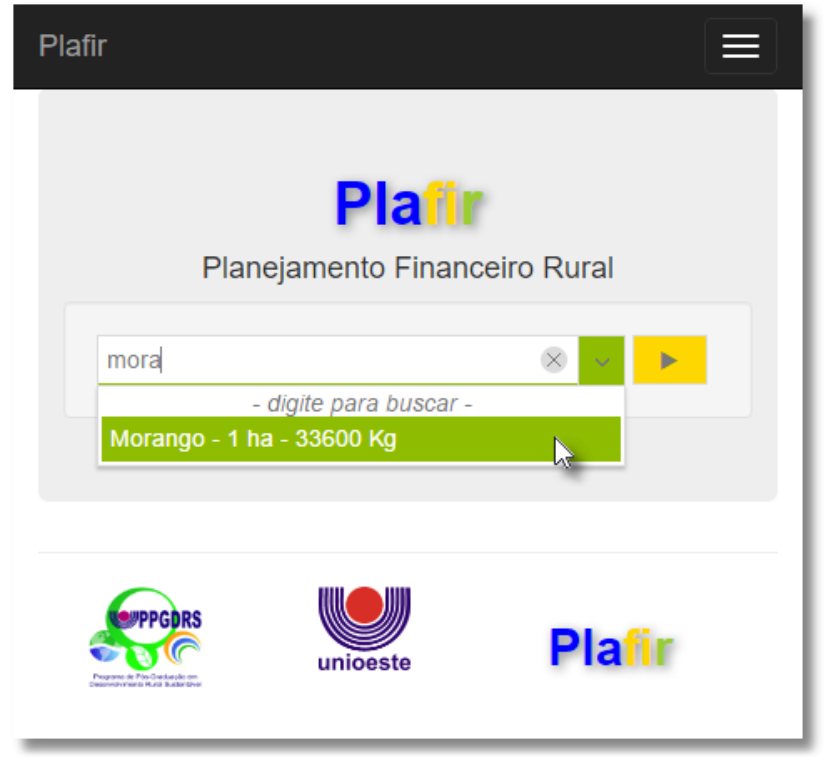

Figura 3. Tela inicial com localização de produto

Fonte: Dados da pesquisa (2016)

Este buscador mostra modelos previamente cadastrados por um técnico agrícola. Quando o agricultor pretende diversificar sua produção, nem sempre possui conhecimento sobre os insumos necessários para uma nova atividade rural. Desta maneira, é necessária a intervenção de um técnico agrícola para que insira, no Plafir, modelos financeiros de produção e mantenha atualizados os preços de insumos. Assim, o aplicativo se torna atraente para o produtor rural realizar simulações de um produto de seu interesse. Esta tela inicia o processo descrito no diagrama de sequência D1 e faz referência ao requisito F1.

Após selecionar o modelo desejado (requisito F3) e confirmar a operação, uma nova página será carregada com os dados do modelo, porém com valores dos itens atualizados de acordo com o preço vigente (requisito F4). Mesmo que o modelo tenha sido registrado há tempos, com os preços defasados, sempre que realizar a operação de simulação, o Plafir buscará preços mais recentes.

Inicialmente, o agricultor deverá parametrizar sua produção, identificando a área produtiva em hectares. Ao alterar a quantidade de área, o Plafir recalculará a proporção equivalente à produção estimada e a quantidade de todos os itens inseridos no planejamento, conforme a Figura 4. 


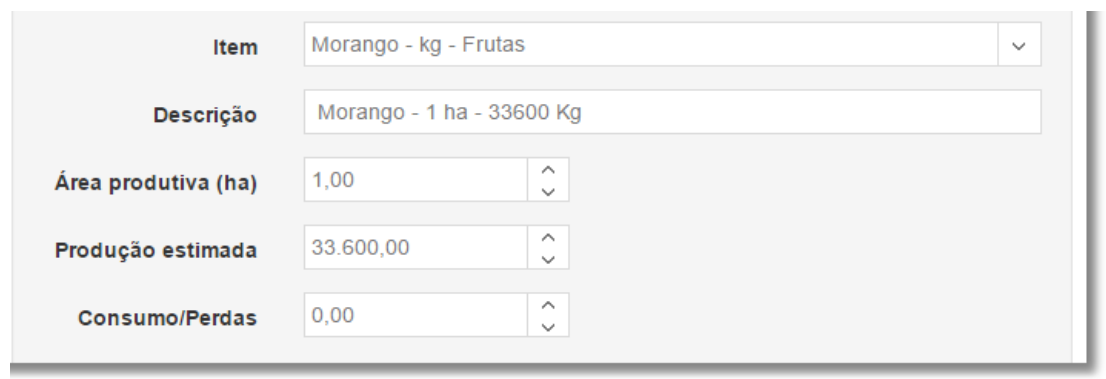

Figura 4. Parâmetros do simulador financeiro

Fonte: Dados da pesquisa (2016)

Outros dados são solicitados, além da área produtiva e produção estimada. Assim, o campo "item" representa o produto a ser planejado; a descrição permite detalhar o planejamento com mais informações acerca da produção; o consumo/perdas calcula a estimativa de descarte do produto.

$\mathrm{Na}$ sequência, os itens que compõem as receitas e despesas do demonstrativo financeiro são apresentados. $\mathrm{O}$ aplicativo permite cadastrar agrupamentos, conforme a necessidade. O planejamento da Figura 5 encontra-se dividido em três grupos: a receita contém itens de venda da produção, enquanto os insumos e serviços contêm itens de custos e despesas.

\begin{tabular}{|c|c|c|c|c|c|c|c|}
\hline Item & Quantidade & Unitário & & Total & \multicolumn{2}{|l|}{$\%$} & \\
\hline \multicolumn{8}{|l|}{ Novo grupo } \\
\hline \multicolumn{6}{|c|}{ Receita } & Alterar & Excluir \\
\hline Venda Feira & $10080 \mathrm{~kg}$ & 6,75 & $\$$ & $68.040,00$ & $32,85 \%$ & Alterar & Excluir \\
\hline Venda Cooperativa & $23184 \mathrm{~kg}$ & $* 6,00$ & $\$$ & $139.104,00$ & $67,15 \%$ & Alterar & Excluir \\
\hline \multicolumn{8}{|l|}{ Novo item } \\
\hline \multicolumn{8}{|c|}{ R\$ $207.144,00100,00 \%$} \\
\hline \multicolumn{6}{|c|}{ Insumos } & Alterar & Excluir \\
\hline Adubo & 1 ha & $1.200,00$ & $\$$ & $1.200,00$ & $1,57 \%$ & Alterar & Excluir \\
\hline Caixas de Papelão & 20000 und & 0,50 & $\$$ & $10.000,00$ & $13,08 \%$ & Alterar & Excluir \\
\hline Inseticidas & 261 & 200,00 & $\$$ & $5.200,00$ & $6,80 \%$ & Alterar & Excluir \\
\hline Fertilizante & 1001 & 3,00 & $\$$ & 300,00 & $0,39 \%$ & Alterar & Excluir \\
\hline Muda de morango & 50000 und & 0,25 & $\$$ & $12.500,00$ & $16,35 \%$ & Alterar & Excluir \\
\hline \multicolumn{8}{|l|}{ Novo item } \\
\hline & & & & $2 \$ 29.200,00$ & $38,20 \%$ & & \\
\hline \multicolumn{6}{|c|}{ Serviços } & Alterar & Excluir \\
\hline Distribuição adubos & $12 \mathrm{~d} / \mathrm{h}$ & 60,00 & $\$$ & 720,00 & $0,94 \%$ & Alterar & Excluir \\
\hline Aplicação fertilizantes & $12 \mathrm{~d} / \mathrm{h}$ & 60,00 & $\$$ & 720,00 & $0,94 \%$ & Alterar & Excluir \\
\hline Aplicação defensivos & $20 \mathrm{~d} / \mathrm{h}$ & 60,00 & $\$$ & $1.200,00$ & $1,57 \%$ & Alterar & Excluir \\
\hline Desbrota e limpeza & $80 \mathrm{~d} / \mathrm{h}$ & 60,00 & $\$$ & $4.800,00$ & $6,28 \%$ & Alterar & Excluir \\
\hline Irrigação & $5 \mathrm{~d} / \mathrm{h}$ & 60,00 & $\$$ & 300,00 & $0,39 \%$ & Alterar & Excluir \\
\hline Plantio & $40 \mathrm{~d} / \mathrm{h}$ & 60,00 & $\$$ & $2.400,00$ & $3,14 \%$ & Alterar & Excluir \\
\hline Aração & $5 \mathrm{~h} / \mathrm{m}$ & 110,00 & $\$$ & 550,00 & $0,72 \%$ & Alterar & Excluir \\
\hline Gradagem & $5 \mathrm{~h} / \mathrm{m}$ & 110,00 & $\$$ & 550,00 & $0,72 \%$ & Alterar & Excluir \\
\hline Colheita/Classificação & $600 \mathrm{~d} / \mathrm{h}$ & 60,00 & $\$$ & $36.000,00$ & $47,10 \%$ & Alterar & Excluir \\
\hline \multicolumn{8}{|l|}{ Novo item } \\
\hline & & & & $2 \$ 47.240,00$ & $61,80 \%$ & & \\
\hline
\end{tabular}

Figura 5. Dados simulados do planejamento financeiro

Fonte: Dados da pesquisa (2016)

Os valores do orçamento são demonstrados em formato de tabela. Cada linha apresenta a descrição do item, a quantidade do produto, o valor unitário, o total e o percentual equivalente à proporção dentro de receitas/despesas. Esta análise vertical permite ao agricultor identificar quais itens terão maior participação monetária no planejamento.

Junto ao valor unitário de cada item está presente um botão com a simbologia "\$", que possibilita acompanhar as variações de preço de cada item por meio de uma representação gráfica, em formato de linha, conforme a Figura 6. 


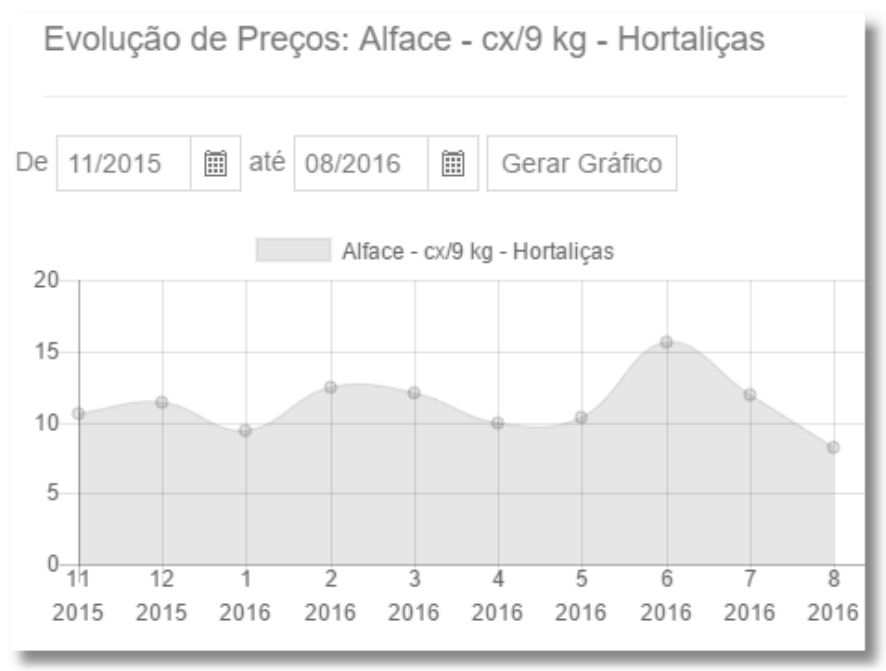

Figura 6. Gráfico de evolução de preços de um item

Fonte: Dados da pesquisa (2016)

Desse modo, o produtor rural pode informar o mês inicial e final de consulta e obter os preços do período. Ao selecionar um determinado ponto no gráfico, surgirá uma caixa com o preço daquela competência. Este gráfico faz referência ao requisito F5.

Para cada item orçado no planejamento, é possível alterar as quantidades e o valor unitário. Além disso, é possível excluir itens ou incluir novos. Sempre que algum valor for manipulado, o aplicativo recalculará o resultado automaticamente em tempo real, conforme a Figura 7.

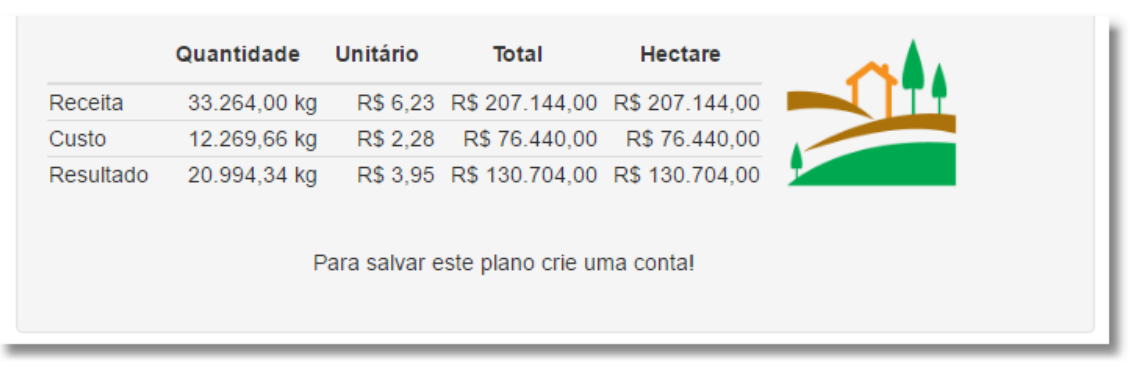

Figura 7. Resultado do planejamento financeiro

Fonte: Dados da pesquisa (2016)

Os dados resultantes do planejamento estão localizados em uma tabela logo abaixo dos itens orçados. Um dos dados se refere à estimativa proveniente da venda do produto. A tabela apresenta, ainda, a quantidade estimada de uma possível venda, o valor unitário bruto, o total monetário da receita e o valor da receita por hectare. Em seguida, permite acompanhar dados referentes ao custo da produção como o ponto de equilíbrio em quantidade, ou seja, quanto seria necessário vender para pagar as despesas, além do custo unitário do produto, do custo total da produção e do valor do custo por hectare. Por fim, o resultado do planejamento apresenta a diferença entre as variáveis correspondentes à linha da receita, confrontadas com as da linha de custo. O resultado final corresponde à margem bruta do produto (Figura 7).

A simulação do planejamento financeiro pode ser salva mediante um cadastro do agricultor no sistema. Este permite a inclusão, alteração, consulta e exclusão de planos, conforme o requisito F2. Depois da identificação do agricultor no aplicativo, será possível consultar os insumos disponíveis no sistema, bem como o histórico de preços.

Outro cadastro necessário no Plafir é o do técnico agrícola. Uma vez identificado o perfil, o aplicativo possibilitará cadastrar modelos de planejamento. Estes são disponibilizados para todos os usuários técnicos agrícolas, conforme o requisito F3. Desta maneira, todos poderão manter os modelos íntegros e os preços de insumos atualizados (requisito F4). 
Assim, o Sistema de Informação resultante deste projeto, o Plafir, auxiliará na tomada de decisão quando o agricultor desejar investir em uma nova atividade rural, considerando-se:

a) O interesse do agricultor na diversificação;

b) A dificuldade no planejamento financeiro e tomada de decisão para nova atividade rural;

c) A falta de aplicativos atualizados e intuitivos;

d) O interesse por ferramentas de auxílio nestes aspectos; e

e) A disponibilidade do acesso à Internet.

\section{Conclusão}

Os agricultores familiares podem usufruir dos benefícios do Plafir para planejar novas atividades rurais, pois o aplicativo provê subsídios à tomada de decisão por meio de indicadores financeiros. $\mathrm{O}$ rol de insumos que compõe o custo variável contém quantidades relevantes para melhor compreensão da atividade. Os resultados financeiros são de fácil entendimento e norteiam a decisão. O aplicativo é intuitivo e chega-se ao resultado em poucos cliques.

Um dos limitantes do projeto é a diferenciação de preços por localidade. O aplicativo não disponibiliza o local dos preços de insumos, sendo necessário que o Plafir esteja disponível apenas para a utilização em uma região. Outro limitante é a dificuldade de se obter e lançar os preços de insumos, pois depende da intervenção do técnico agrícola para que frequentemente atualize o preço dos insumos.

As recomendações futuras e a continuidade do projeto estão baseadas nas limitações citadas. Desse modo, recomenda-se identificar a localidade do agricultor e que os preços sugeridos sejam obtidos dentro de um raio mínimo. Propõe-se, ainda, uma ação junto ao poder público a fim de normatizar um protocolo de disponibilização de preços médios de insumos. Soma-se a isso que algumas organizações promovem ações diárias de levantamento de custos e tais dados poderiam alimentar o Plafir de forma automatizada.

\section{Referências}

AIAF. Agricultura familiar: por um sistema alimentar sustentável. Disponível em <http://www.aiaf2014.gov.br/sitemda/sites/site aiaf/files/argumentos e dados af no mundo - aiaf 2014.pdf> Acesso em: 15 out. 2014.

BEZERRA, Eduardo. Princípios de Análise e Projeto de Sistema com UML. 3. ed. Rio de Janeiro: Elsevier, 2015.

CHAMBERS, James; PAQUETTE, David; TIMMS, Simon. ASP.NET core application development: building an application in four sprints. Redmond, Washington: Microsoft Press, 2017.

COMPANHIA NACIONAL DE ABASTECIMENTO (CONAB). Custos de produção agrícola: a metodologia da Conab. Brasília: CONAB, 2010. Disponível em: <http://www.conab.gov.br/conabweb/download/safra/custos.pdf>. Acessoem: 16 jan. 2017.

CORONEL, Carlos; MORRIS, Steven. Database systems: design, implementation, and management. 12. ed. Boston, MA, USA: Cengage Learning, 2017.

ELLIS, Frank. Rural livelihoods and diversity in developing countries. Oxford: Oxford University Press, 2000.

FAO. Agricultura familiar. Disponível em: <http://www.fao.org/family-farming-2014/pt/>. Acesso em: 20 out. 2014.

FOWLER, Martin. UML Essencial: um breve guia para linguagem-padrão de modelagem de objetos. 3. ed. Porto Alegre: Bookman, 2007.

FREEMAN, Adam. Pro ASP.NET Core MVC. 6. ed. Berkeley, CA: Apress, 2016.

GUANZIROLI, Carlos; ROMEIRO, Ademar; BUAINAIN, Antonio M.; DI SABBATO, Alberto; BITTENCOURT, Gilson. Agricultura familiar e reforma agrária no século XXI. Rio de Janeiro: Garamond, 2001. 
GUEDES, Gilleanes T. A. UML 2: guia prático. 2. ed. São Paulo: Novatec, 2014.

GUZMÁN, Eduardo Sevilla. Origem, evolução e perspectivas do desenvolvimento sustentável. In: ALMEIDA, Jalcione; NAVARRO, Zander (orgs.) Reconstruindo a agricultura: idéias e ideais na perspectiva de um desenvolvimento rural sustentável. Porto Alegre: Editora da Universidade - UFRGS, 1998.

IAP. Módulos fiscais do município do estado do paraná. Governo do Paraná. Disponível em: <http://www.iap.pr.gov.br/pagina1328. html>. Acesso em: 15 jul. 2016.

IBGE. Censo agropecuário 2006: resultados preliminares. IBGE, 2006.

IBGE. Pesquisa nacional por amostra de domicílios: síntese de indicadores 2014. Rio de Janeiro: IBGE, 2015.

LAUDON, Kenneth; LAUDON, Jane. Sistemas de informação gerenciais. 9. ed. São Paulo: Pearson Prentice Hall, 2010.

MARION, José Carlos. Contabilidade rural: contabilidade agrícola, contabilidade da pecuária. 14. ed. São Paulo: Atlas, 2014.

MENDES, Cássia Isabel Costa; OLIVEIRA, Deise R. M. dos Santos; SANTOS, Anderson Rodrigo dos. Estudo do mercado brasileiro de software para o agronegócio. Campinas: Embrapa Informática Agropecuária, 2011.

NAGEL, Christian. Professional C\# 6 and .NET Core 1.0. Indianapolis, IN: Wiley, 2016.

O'BRIEN, James A. Sistemas de informação e as decisões gerenciais na era da internet.2. ed. São Paulo: Saraiva, 2004.

OBE, Regina O.; HSU, Leo S. PostgreSQL: up and running: a practical introduction to the advanced open source database. 2. ed. Sebastopol, CA: O'Reilly Media, 2014.

PRICE, Mark J. C\# 6 and .NET Core 1.0 modern cross-platform development: create powerful cross-platform applications using C\# 6, Net Core 1.0 ASP.NET Core 1.0 and Visual Studio 2015. Birmingham, UK: Packt Publishing, 2016.

RIGGS, Simon et al. PostgreSQL 9 administration cookbook: over 150 recipes to help you run an efficient PostgreSQL database in the cloud. 2. ed. Birmingham, UK: PacktPublishing, 2015.

SACHS, Ignacy. Estratégias de transição para o século XXI: desenvolvimento e meio ambiente. São Paulo: Studio Nobel, 1993

SANTOS, Gilberto José dos; MARION, José Carlos; SEGATTI, Sonia. Administração de custos na agropecuária. 4. ed. São Paulo: Atlas, 2009

SCHNEIDER, Sergio. Rural development through rural livelihood diversification: an overview of Brazilian experience. In: International Conference Dynamics of Rural Transformations in Emerging Economies. 2010. p. 14-16.

SORDI, José Osvaldo de; MEIRELES, Manuel. Administração de sistemas de informação: uma abordagem interativa. São Paulo: Saraiva, 2010.

WAZLAWICK, Raul Sidnei. Análise e design orientados a objetos para sistemas de informação: Modelagem com UML, OCL e IFML. 3.ed. Rio de Janeiro: Elsevier, 2015. 


\section{Dados dos autores}

Luciano Lizzoni

Mestre em Desenvolvimento Rural Sustentável pela Unioeste (2017). Especialista em Tecnologia Java pela União Pan-Americana de Ensino (2008). Graduado em Sistemas de Informação pela Faculdade Sul Brasil (2006).

lizzoni@gmail.com

\section{Aldi Feiden}

Doutor em Ecologia de Ambientes Aquáticos Continentais pela Universidade Estadual de Maringá (2003). Mestre em Ecologia de Ambientes Aquáticos Continentais pela Universidade Estadual de Maringá (1999). Graduado em Agronomia pela Universidade Estadual de Maringá (1989).

aldifeiden@gmail.com

\section{Armin Feiden}

Doutor em Agronomia (Energia na Agricultura) pela Universidade Estadual Paulista Júlio de Mesquita Filho (2001). Mestre em Engenharia Florestal (Conservação da Natureza) pela Universidade Federal do Paraná (1994). Graduado em Agronomia pela Universidade Federal do Paraná (1986). Graduado em Zootecnia pela Universidade Estadual de Maringá (1981).

armin.feiden@gmail.com

Recibido - Received: 2017-04-10

Aceitado - Accepted: 2018-12-13

\section{$(\mathrm{cc}) \mathrm{BY}$}

This work is licensed under a Creative Commons Attribution 4.0

United States License.

\section{UILIS DDof}

This journal is published by the University Library System of the University of Pittsburgh as part of its D-Scribe Digital Publishing Program and is cosponsored by the University of Pittsburgh Press. 